\title{
First on-sky results with ARGOS at LBT
}

G. Orban de Xivry ${ }^{\mathrm{a}, \mathrm{b}}$, S. Rabien ${ }^{\mathrm{b}}$, L. Busoni ${ }^{\mathrm{c}}$, W. Gaessler ${ }^{\mathrm{d}}$, M. Bonaglia ${ }^{\mathrm{c}}$, J. Borelli $^{\mathrm{d}}$, M. Deysenroth $^{\mathrm{b}}$, S. Esposito ${ }^{\mathrm{c}}$, H. Gemperlein ${ }^{\mathrm{b}}$, M. Kulas ${ }^{\mathrm{d}}$, M. Lefebvre ${ }^{\mathrm{e}}$, T. Mazzoni ${ }^{\mathrm{c}}$, D. Peter ${ }^{\mathrm{d}}$, A. Puglisi ${ }^{\mathrm{c}}$,W. Raab ${ }^{\mathrm{f}}$, G. Rahmer ${ }^{\mathrm{e}}$, A. Sivitillid ${ }^{\mathrm{d}}$,J. Storm ${ }^{\mathrm{g}}$, and J. Ziegleder ${ }^{\mathrm{b}}$

${ }^{a}$ Space sciences, Technologies and Astrophysics Research Institute, Université de Liège, Allée du Six Août 17, 4000 Liège, Belgium

bMax Planck Institut für extraterrestrische Physik, Giessenbachstrasse 1, 85748 Garching, Germany

${ }^{\mathrm{C} I N A F}$ Osservatorio Astrofisico di Arcetri, L.go E. Fermi 5, 50125 Firenze, Italy

${ }^{d}$ Max-Planck-Institut für Astronomie, Koenigstuhl 17, 69117 Heidelberg, Germany

eLarge Binocular Observatory, North Cherry Avenue 933, Tucson, 85721 Arizona, USA

fEuropean Space Agency, ESTEC, Keplerlaan 1, 2201AZ Noordwijk, The Netherlands

${ }^{g}$ Leibniz-Institut für Astrophysik Potsdam, An der Sternwarte 16, 14482 Potsdam, Germany

\begin{abstract}
One year and an half after ARGOS first light, the Large Binocular Telescope (LBT) laser guided ground-layer adaptive optics (GLAO) system has been operated on both sides of the LBT. The system fulfills the GLAO promise and typically delivers an improvement by a factor of 2 in FWHM over the $4^{\prime} \times 4^{\prime}$ field of view of both LUCI instruments, the two near-infrared imagers and multi-object spectrographs.

In this paper, we report on the first on-sky results and analyze the performances based on the data collected so far. We also discuss adaptive optics procedures and the joint operations with LUCI for science observations.
\end{abstract}

Keywords: Ground-Layer Adaptive Optics; Laser Guide Stars; Large Binocular Telescope

\section{INTRODUCTION}

ARGOS $^{1}$ is the laser guide stars and wavefront sensing facility for the Large Binocular Telescope. By sensing the ground-layer turbulence from three Rayleigh laser guide stars (LGS) forming an asterism of $2^{\prime}$ radius at $12 \mathrm{~km}$ above the telescope, it delivers an improvement by a factor around two in FWHM over the $4^{\prime} \times 4^{\prime}$ field of view (FoV) of both LUCI instruments. ${ }^{2}$ LUCI 1 and LUCI 2 are two near-infrared wide field imagers and multi-object spectrographs which capability and efficiency will be boosted by the increased resolution and encircled energy.

The first on-sky ground-layer adaptive optics (GLAO) loop closure with ARGOs has been achieved in Fall 2014 on the right side of the telescope, while the left side's commissioning on sky has started in December 2015. In short, both ARGOs systems have started to deliver their expected performances while waiting for a scientific demonstration.

Upon its completion and demonstrated efficiency, the combination of LUCI and ARGOS at LBT will become a unique facility: the only 8-m class telescope implementing a GLAO correction combined with near-infrared multiobject spectroscopy. The project will be competitive with WFPC $3^{3}$ on HST, its $\sim 2^{\prime} \times 2^{\prime}$ FoV and $0.135^{\prime \prime} \times 0.121^{\prime \prime}$ pixel scale from $800 \mathrm{~nm}$ to $1700 \mathrm{~nm}$ have to be compared to the $4^{\prime} \times 4^{\prime}$ with $0.12^{\prime \prime} \times 0.12^{\prime \prime}$ pixel scale up to the K-band with LUCI combined with ARGOS.

This paper is for a large part a summary of what has been presented ${ }^{4,5}$ at the last AO4ELT4 conference. In the last six months, we have now also performed GLAO loop closure on the left side and have started to collect a small number statistics of the performance of our system. Those results confirms the early statements and show that ARgos provides a correction oscillating around a factor 2 in PSF FWHM. In the next sections, we first present ARGOS in a nutshell. We then summarize the adaptive optics operations and procedures developed for ARgos. Finally, we present the on-sky results in term of uniformity, sensitivity, and PSF FWHM statistics.

Further author information: (Send correspondence to G. Orban de Xivry)

G. Orban de Xivry: E-mail: gorban@ulg.ac.be, Telephone: (0032) 43669712

Adaptive Optics Systems V, edited by Enrico Marchetti, Laird M. Close, Jean-Pierre Véran, Proc. of SPIE Vol. 9909,990936 (C) 2016 SPIE · CCC code: 0277-786X/16/\$18 · doi: 10.1117/12.2240094 


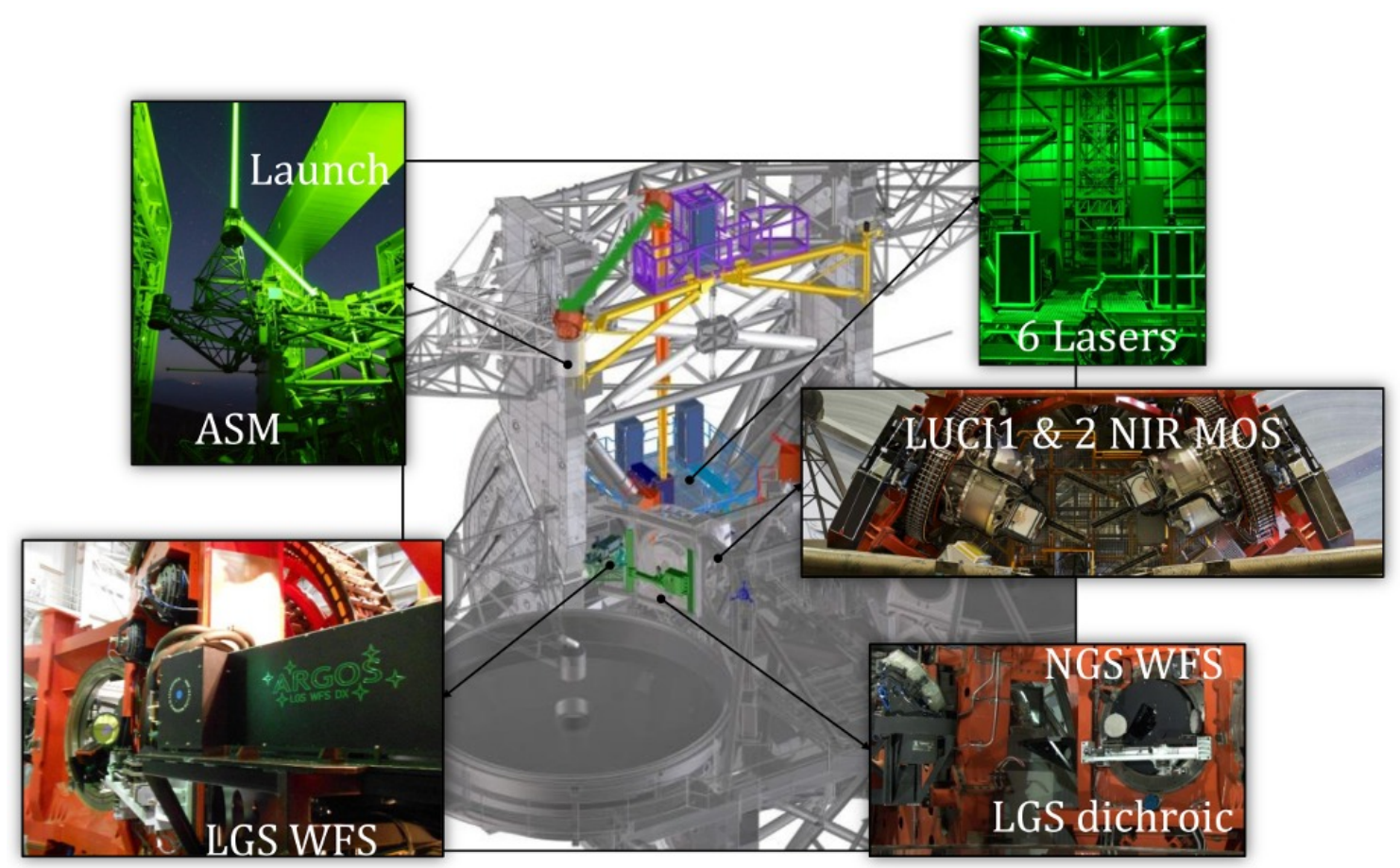

Figure 1. Illustration of the ARgos components and how they are distributed over the telescope structure. The three laser launch is done on-axis behind the secondary mirror. The NGS board sits in front of the LUCI instrument inside the de-rotator, while the LGS light is directed by a dichroic system (dichroic plus mirror) towards the LGS wavefront sensor.

\section{SYSTEM SUMMARY}

A detailed description of the system, the design and development of the Argos subsystems is presented in several proceedings : general presentation of the system, ${ }^{1,4}$ the LGS wavefront sensor,${ }^{6-9}$ the laser and launch system, ${ }^{10-12}$ and the control software. ${ }^{13,14}$ We present here in a nutshell the working principle of ARGOs.

To fullfill its objectives and improve by a factor of two in FWHM size over the $4^{\prime} \times 4^{\prime}$ FoV, Argos features three pulsed Rayleigh beacons per each side of the LBT. The backscattered photons are detected by ShackHartmann based wavefront sensors. Those sensors include the acquisition and stabilization of the laser spots, the gating of the required guide star height with Pockels cells and the imaging of all three Shack-Hartmann pupils on a single large frame CCD. Those measurements then feed our wavefront sensor real-time computer which is interfaced to the adaptive secondary mirror computers. In addition, the system is composed of : a quad-cell based on avalanche photo-diodes tip-tilt sensor to allow the faintest tip-tilt guide stars, a dedicated calibration system also used for daytime operation, and all the necessary instrument control software.

An illustration of the sub-systems and how they spread all over the telescope is presented in Figure 1.

Argos fully exploits the provided active and adaptive modules of the LBT. In particular, ArGos relies on the $0.94 \mathrm{~m}$ adaptive secondary mirrors (ASM) conjugated at $150 \mathrm{~m}$ above ground to perform its correction. The adaptive module also includes a real time computer (RTC) that is used by every AO system at LBT for wavefront reconstruction. Upstream of it, the ARGOS RTC acts as a framegrabber, slope computer, piezo controller for the LGS jitter stabilization loops, and collector of the slopes from the quad-cell tip-tilt sensor and from the First Light Adaptive Optics (FLAO) pyramid wavefront sensor. It finally assembles the full ARGOs slope vector that is sent to the ASM RTC for modal reconstruction and control of the ASM. 


\section{ADAPTIVE OPTICS OPERATIONS}

We present in this section the full chain of operations of ARGOs in chronological order. More details on the adaptive optics procedures and the choices of those specific solutions can be found in 5 .

1. Telescope collimation. The current chosen scheme consists in a dedicated preset to collimate the telescope on-axis through the ARGOS dichroic on a bright pointing star in the proximity of the scientific target $\left(<1^{\circ}\right)$. This allows to quickly collimate the telescope, with the additional benefit of a simultaneous update of the telescope pointing model. After the collimation, a new preset is sent that slews the telescope to the scientific target in tracking mode, in parallel moving the guider probe away from the LuCI field. Since the repointing is small the telescope collimation is preserved and adaptive optics operations can be immediately started. The additional time spent for slewing the telescope from the pointing star to the scientific target is less than a minute and the entire sequence can be fully automatized in the LUCI scripts resulting overall in the fastest and most reliable preset sequence we have found.

2. Laser propagation. Once pointing at the target, the lasers can be propagated on sky. This requires all interlocks to be clear to propagate, including the satellite avoidance system. The laser officer requires clearance for propagation to the two aircraft human spotters. Only then the laser officer opens the propagation shutter and lasers are launched on sky. The typical time required to propagate the lasers on sky is $\sim 2 \mathrm{~min}$. LBT is currently testing an automated detection system based on TBAD* devices ${ }^{15}$ that should speed the sequence.

The next step is the pre-acquisition of the lasers onto the laser wavefront sensor patrol cameras. A laser alignment telescope with a field of $\sim 1^{\circ}$ allows the semi-automatic acquisition of the lasers by analyzing the position of the Rayleigh plumes on sky and steering one of the launch mirrors.

3. Laser beam shaping. Focus of the launch telescope can be adjusted in order to form the images of the laser spots in a plane conjugated to the LGS wavefront sensor (WFS) focal plane. Another effect also impacts the ARgOS LGS spots: the two large borosilicate flat mirrors of the launch system appears to show temperature-dependent bending which introduces mostly focus and astigmatism. This effect depends on the deposited laser power (less than a watt), the wind orientation and the outdoor temperature. To correct this time-varying effect, counter-heaters on the back surfaces of the mirrors have been installed and an astigmatism corrector, composed of two tilted thick $(12.5 \mathrm{~mm})$ plane parallel plates in the diverging beam $(\mathrm{f} \#=20)$, has been introduced. While the heaters are aimed to equilibrate front and back temperatures, the astigmatism corrector allows a more precise and deterministic corrections. This astigmatism correction is optional and most needed at the beginning of the night. It typically requires 2 minutes and is supervised by the ARgOs operator.

4. LGS acquisition \& guiding. After the pre-acquisition on to the LGS WFS patrol camera and the (optional) beam shaping, the lasers are fine-acquired on the WFS within an aperture of $\sim 4.5^{\prime \prime}$. This step is typically done manually where the ARGOS operator has the easy task of identifying the LGS spots (clicking on the 3 patrol camera images) which then drives one of the laser launch mirrors. This acquisition procedure is much more robust in case of thin clouds scattering light on the patrol camera field.

Once acquired, jitter stabilization is automatically started removing any atmospheric tip-tilt or telescope jitter on the WFS by controlling three piezo mirrors at $1 \mathrm{kHz}$ (one for each LGS). A low pass filtered (1Hz) mean position of those piezo mirrors provides the pointing drift of the LGS asterism. This drift is corrected by a pupil mirror in the launch optics controlling the position on sky, with high accuracy but limited range $\left(20^{\prime \prime}\right.$ on sky). The pupil mirror itself is periodically offloaded to a launch mirror in maximum steps of $0.5^{\prime \prime}$.

5. Natural Guide Star (NGS) acquisition. In parallel to the LGS acquisition, the NGS sensor ${ }^{\dagger}$ is configured based on the natural star position and magnitude as provided by the LUCI preset : the NGS board is moved to the star position and, based on a look-up table, it configures the pyramid WFS camera (framerate, binning) and the filter wheels that split the light between the two arms of the NGS board (splitting

\footnotetext{
${ }^{*}$ Transponder Based Aircraft Detector.

${ }^{\dagger}$ the First Light Adaptive Optics board into which has been integrated the ArGos quad-cell tip-tilt sensor.
} 
light between tip-tilt and truth sensing). Once configured, the NGS appears in the NGS technical viewer $\left(14^{\prime \prime} \mathrm{FoV}\right)$, the position of the star is automatically determined and the NGS board is moved to fine-center the NGS on a previously determined target pixel of the technical viewer. A second filter wheel is then moved to its mirror position to send the light to the tip-tilt quad-cell sensor.

6. Adaptive optics control loop. Once the NGS acquisition is completed and the LGS acquisition has been confirmed by the ARGOS operator, the adaptive optics loop is automatically closed and consists in the following steps :

- Loading the AO reconstructor to the adaptive secondary mirror (ASM). The reconstructor is selected in a lookup table based on the Shack-Hartmann sensor configuration and its slope algorithm, and the mode of tip-tilt correction (none, quad-cell, or pyramid).

- Closing the tip-tilt loop at $100 \mathrm{~Hz}$ and wait for the offload from the ASM to the hexapod mount.

- Co-pointing of the quad-cell and the pyramid. Since residual mis-alignment is present and it depends on the rotator angle (the pyramid being re-rotated by a K-mirror while the quad-cell is not), this loop is kept running as low frequency. It allows the truth sensing to perform in optimal conditions.

- Adjusting the integration time of the tip-tilt quad-cell sensor. Based on the measured counts, the magnitude is estimated and the integration time (thus the tip-tilt loop speed) is adapted following a similar but simplified approach to 16 , between $100 \mathrm{~Hz}$ and $1 \mathrm{kHz}$.

- Offload focus to the time-of-flight. In order to maximize the dynamical range of the LGS WFS and to avoid a sudden too large focus command when the LGS loop is closed, we first minimize the LGS WFS focus term by modifying the time delay between the laser pulse and the gating opening time.

- In three steps, we ramp up the mode gain while waiting for offload of the ASM to the hexapod in between each step.

- The truth sensing loop is started. The "true" wavefront error of the first $\sim 20$ modes is measured by the pyramid and converted to LGS WFS signals through a multiplication by the LGS interaction matrix. Focus is offloaded to the time-of-flight while the other modes are projected on the LGS slope offsets.

- Optimization of the gain. As a last step, the modal gain can be optimized. This is done by scanning a range of modal gains and searching for the values that minimize the WFS signal variance. The gains are typically divided in three group : tip-tilt, low order from 2 to 36 , high order up to 150 . They can also be tweaked independently by the operator.

At the end of this sequence, requiring about 1 minute, the LUCI observation begins.

7. Managing dithering, offsets and asynchronous interruptions. During the execution of the LUCI observing block, different events require pausing the adaptive optics correction for a short period, namely : small offsets $<15^{\prime \prime}$ (typically dithering), large offset $>15^{\prime \prime}$ (e.g. sky pointings), and satellite or aircraft interruptions.

In case of small offsets, the laser propagation is not interrupted : since the launch telescope is part of the telescope, the LGS spots remain centered on the WFS field and the loop does not need to be paused. The NGS loop, of course, needs to be paused by setting the gain of the modes controlled by the NGS to zero. The NGS board is then offset to recenter the NGS star on the tip-tilt sensor and the NGS loop is automatically resumed by ramping up in two steps the relevant modal gain. The full process is transparent to the ARGos operator and does not generate any significant overhead.

For larger offset, the laser propagation is briefly interrupted and resumed when the slew is terminated. In this case, both LGS and NGS loops are paused and resumed once the lasers are back on sky.

Finally the laser closed-loop operation can be interrupted by satellite or aircraft. In the case of satellite, the control software attempts to pause the LGS loop before stopping the lasers to ensure safe reaction and to allow to resume quickly the closed-loop operation once the laser are back on-sky. The loop is resumed manually : to operator has to reconfirm the LGS acquisition (or reacquire them if necessary). For feedbacks on those interruptions at LBT, see 17. 

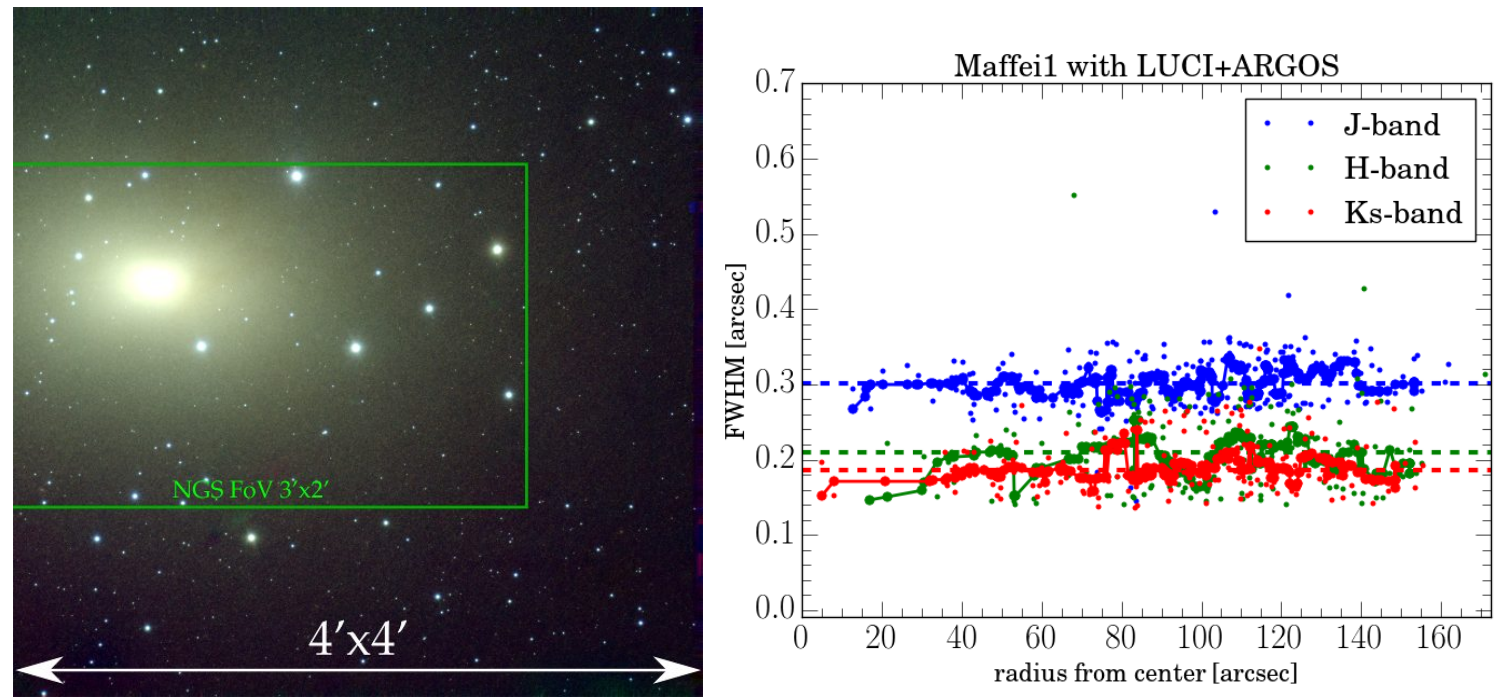

Figure 2. (Left) Maffeil observation in $\mathrm{J}, \mathrm{H}$ and $\mathrm{Ks}$ broad band for a total of 7.5 minute exposure time. The field of view span $4^{\prime} \times 4^{\prime}$ and show a uniform resolution all over. In green is highlighted the field-of-view available to pick a NGS for tip-tilt and truth sensing correction. (Right) PSF FWHM as a function of radius from the center of the FoV, where was also the tip-tilt NGS. The constant distribution shows that there is no apparent sign of anisoplanatism and that the ArgOs GLAO correction is very uniform.

\section{FIRST ON-SKY RESULTS}

First ARGOS commissioning results obtained on the right side of LBT have been presented in 4. We repeat some of the discussion made there and complement it with new comparisons of ARGos performances and simulations, as well as a larger set of ARGOS on-sky measurements both with left and right sides of LBT. Those results confirms the satisfactory performances of ARGOS providing an improvement in PSF FWHM of a factor around 2 depending on the wavelength, relative strength of the ground-layer, etc..

As a GLAO system, the improvement brought by ARGOS are the increased sensitivity and the uniform improved resolution over a $4^{\prime} \times 4^{\prime}$ field-of-view. In the following sections, we therefore discuss those aspects.

\subsection{Uniformity}

Uniformity of the PSF allows to more easily derive the completeness limits of deep imaging surveys and ease accurate photometry and astrometry. It is also an important parameter in efficiently carrying multi-object spectrograph observations.

ARGOS typically correct 150 modes with tip-tilt being corrected by one NGS and all other modes by performing a tomographic ground-layer reconstruction. The single tip-tilt star is picked in a field-of-view of $2^{\prime} \times 3^{\prime}$ within the LUCI FoV, and is typically taken as close to the center of the field as possible. From this concept, the main potential source of non-uniformity due to the AO system is the tip-tilt anisoplanatism. But since the GLAO correction is modest, the sensitivity to anisoplanatism is also smaller.

The measurements done so far with ARgos and LuCI show no sign of anisoplanatism. Of course this also depends on the location of the tip-tilt star which has been preferentially chosen in the center of the LUCI FoV. This is illustrated in Figure 2, for Maffei1 observed on the left LBT side, showing the constant PSF FWHM as a function of radius for the three NIR bands in closed loop.

\subsection{Point source sensitivity}

An important outcome of improving the resolution is to increase the point source sensitivy. We reproduce here a discussion presented in 4 . 

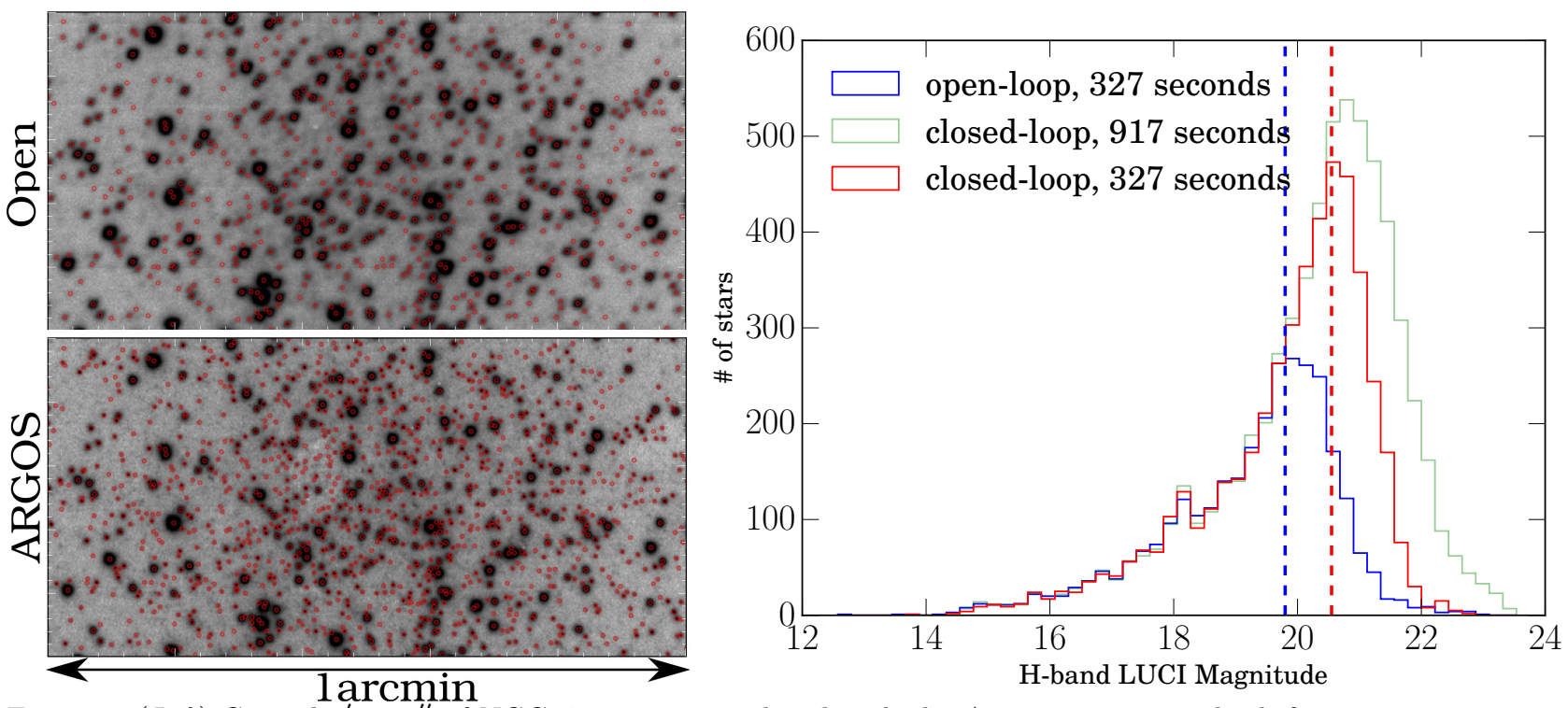

Figure 3. (Left) Central $1^{\prime} \times 30^{\prime \prime}$ of NGC2419 uncorrected and with the ARGos correction, both for 5 minute exposure. Square root stretched intensity scale. Overlaid in red circles are the detected stars with DAOPHOT whose photometry is used for the plot on the right side. (Right) Luminosity function of NGC2419 for three different images 5 minute uncorrected (blue) and with Argos (red), and 15 minutes with Argos. The vertical lines indicate the peak of the distributions of the 5 minute exposure AO corrected and uncorrected. They are separated by $\sim 0.75$ magnitude which is in line with the expected improvement brought by Argos. Credit: 4.

In the background limited regime, for a point source and fixed flux, the signal-to-noise ratio scales as

$$
\frac{\mathrm{S}}{\mathrm{N}} \propto \frac{f_{\text {aper }} t_{i}}{\sqrt{d_{\text {aper }}^{2} t_{i}}},
$$

where $f_{\text {aper }}$ is the fraction of the source flux into the aperture (or slit), $d_{\text {aper }}$ is the diameter of the aperture, and $t_{i}$ is the integration time. If the PSF size is reduced by a factor 2 , for a fixed fraction of the source flux $f_{a p e r}$, the diameter of the aperture can be divided by 2. It results that the $\mathrm{S} / \mathrm{N}$ is itself multiplied by two for a fixed integration time, equivalent to a delta of 0.75 mag.

To illustrate this improvement, we performed PSF photometry on the globular cluster NGC2419 using the standard package DAOPHOT ${ }^{18}$ in PYRAF. The PSF model consists of an analytical profile with additional quadratic variations across the FoV; which provides an adapted model for GLAO data. The PSF is constructed based on a large set of stars (20 to 40 in our case) spread over the FoV. The full photometry process is performed on three data sets : 5 minute exposure uncorrected and AO corrected, and 15 minute exposure AO corrected. The results are illustrated in Figure 3 showing the open-loop and closed-loop images on the 5 minute exposure of the central $1^{\prime} \times 30^{\prime \prime}$. The identified stars are highlighted in red circles and the luminosity functions for the full $4^{\prime} \times 4^{\prime}$ FoV is represented on the right side. By taking the peaks of the luminosity function distributions as an indicator of the sensitivity, we can see that the closed-loop 5 minute exposure reaches a higher sensitivy by approximately 0.75 magnitude compared to the open-loop data. However other effects are folded in, such as the reduced crowding noise that should increased further the sensitivity. On the other hand, the gain in PSF FWHM for this data set is slightly lower than 2 and the PSF shape is also slightly modified (hence the gain in PSF FWHM does not translate in the exact same gain in encircled energy).

A big part of the ARGOS and LuCI science case is multi-object spectroscopy. The argument exposed above is almost directly applicable to spectroscopy where one can select a narrower slit thus decreasing the background noise for a fixed source flux. A narrower slit also allows to reach higher spectroscopic resolution for a same signal-to-noise ratio which is advantageous to reduce the contamination from the numerous atmospheric $\mathrm{OH}$ lines in the near-infrared. 


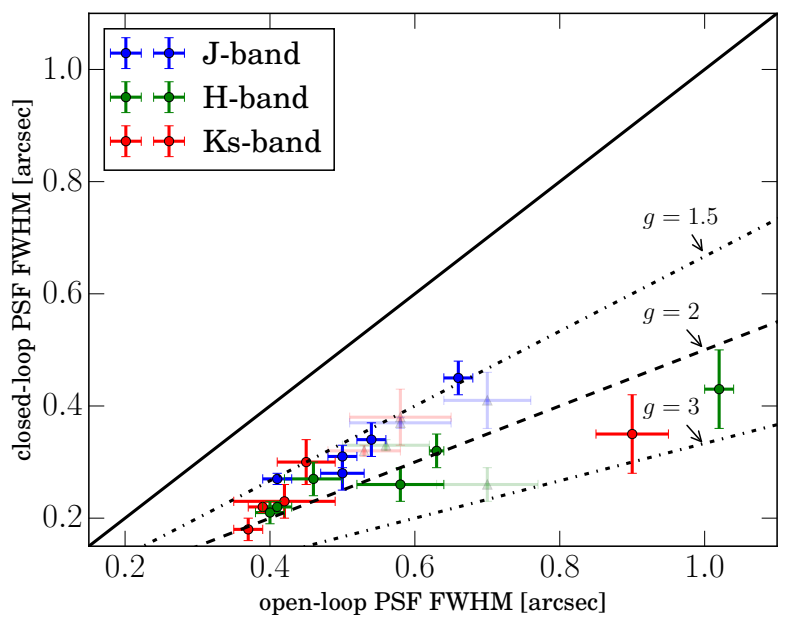

Figure 4. Open-loop vs. closed-loop PSF FWHM comparison in the three broad bands - J, H, Ks - measured on commissioning data on both left and right side. The gain in resolution provided by Argos is consistently around 2 in band $\mathrm{H}$ and $\mathrm{Ks}$, while closer to 1.5 in band J. The open-loop resolution is measured on sky pointing taken in between closed-loop pointings in typical observing sequences Object-Sky-Object.

\subsection{Performance statistics}

The improvement in PSF FWHM sizes has been systematically analyzed on the relevant imaging data acquired so far with ARgos and LucI. To do so, we automatize the point source detection and fit 2D elliptical Moffat profiles with varying power law index and ellipticity.

In most cases, the PSF fits have been performed on individual dithered images, taking the spatial median value as the PSF resolution for a given time. This is run on all closed-loop images (for one target and filter) and the temporal median is taken. The spatio-temporal median values are the numbers presented here. The error is taken as the standard deviation of the temporal sequence. For the open-loop FWHM, we use sky images typically taken in between target observations in a classical Object-Sky-Object observation sequence. The same fit analysis is performed on those sky images and provides our open-loop measurements.

The results are shown in Figure 4 for all three NIR J, H, Ks bands and left and right LBT side. The obtained performances vary from 1.5 to 3 in PSF FWHM gains. As a tentative interpretation, J-band performances seem to be closer to a gain of 1.5 while $\mathrm{H}$ and Ks band are closer to a factor 2 .

Of course several effects are folded into those measurements such as a varying seeing between closed-loop and open-loop, or a varying relative ground layer contribution to the atmospheric turbulence. As part of the ARGOS effort, we are also implementing a SLODAR measurement based on the correlation of the three Shack-Hartmann sensors. The routine is still under test and development, see 19 for more details, but the first results show a direct link between reduced correction gain and reduced relative strength of the ground layer.

Using data taken in good seeing conditions, we compare our performance measurements to simulations done during the Argos design phase, see Figure 5. The plot compares open-loop (blue) and closed-loop (green) in $\mathrm{J}, \mathrm{H}, \mathrm{Ks}$ bands for a $0.8^{\prime \prime}$ seeing. The ARGOS on-sky results are close to the early expectations, in some cases going below the Nyquist sampling limit of the N3.75 camera of LUCI.

\section{CONCLUSION}

Both left and right ARGos are now in operation and reach the expected performances on sky, i.e. an improvement of a factor of about 2 in FWHM over the $4^{\prime} \times 4^{\prime}$ FoV. A large part of the commissioning effort is being spent on optimizing the adaptive optics procedure to minimize the ARGOS operation overheads. Improvements are still in development to make the ARGOS control software as user-friendly, safe and reliable as possible reducing the number of operators needed and maximizing the scientific return of ARGos. 


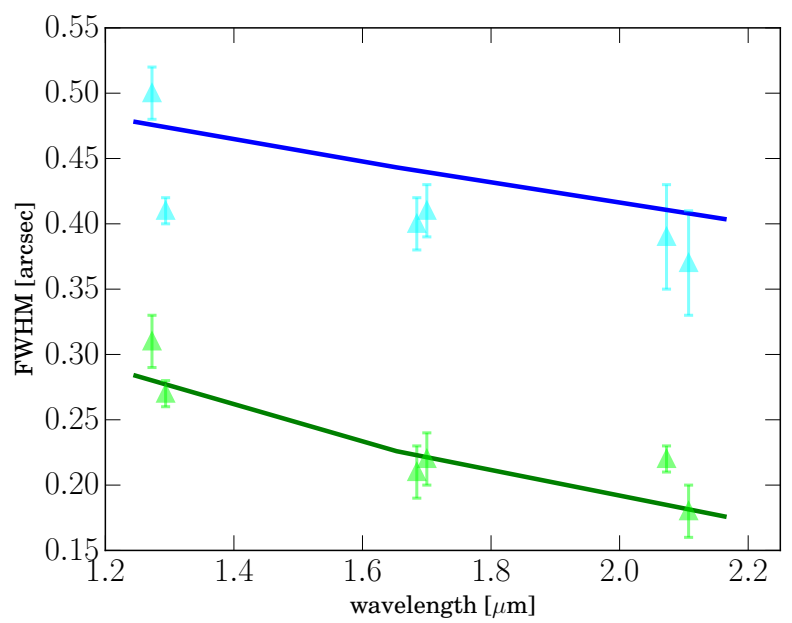

Figure 5. Comparison of early simulations with observations for the three bands : J, H, and Ks. Blue indicates uncorrected observations or simulations. Green indicates a correction with ARGOs. The lines represent simulations in the three broad bands with outer layer $L_{0}=30 \mathrm{~m}$ and seeing of $0.8^{\prime \prime}$.

Multi-object spectroscopic observations are on-going and will be presented in future ArGos publications. They will be the first MOS data acquired on a 8m class telescope with GLAO correction making ARGOS and LUCI a unique facility.

\section{ACKNOWLEDGMENTS}

This research has made use of NASA's Astrophysics Data System Bibliographic Services, of Astropy, a communitydeveloped core Python package for Astronomy, ${ }^{20}$ and of DAOPHOT. ${ }^{18}$ The Argos team wishes to thank the entire LBT telescope crew, telescope managers and telescope operators for their continuous support and dedication to the ARGOS project. Last but not least, special thanks to the airplane spotters standing in the cold and windy Mt Graham nights.

\section{REFERENCES}

[1] Rabien, S., Barl, L., Beckmann, U., Bonaglia, M., Borelli, J. L., Brynnel, J., Buschkamp, P., Busoni, L., Christou, J., Connot, C., Davies, R., Deysenroth, M., Esposito, S., Gässler, W., Gemperlein, H., Hart, M., Kulas, M., Lefebvre, M., Lehmitz, M., Mazzoni, T., Nussbaum, E., Orban de Xivry, G., Peter, D., Quirrenbach, A., Raab, W., Rahmer, G., Storm, J., and Ziegleder, J., "Status of the ARGOS project," in [Society of Photo-Optical Instrumentation Engineers (SPIE) Conference Series], Society of Photo-Optical Instrumentation Engineers (SPIE) Conference Series 9148, 1 (July 2014).

[2] Seifert, W., Appenzeller, I., Baumeister, H., Bizenberger, P., Bomans, D., Dettmar, R.-J., Grimm, B., Herbst, T., Hofmann, R., Juette, M., Laun, W., Lehmitz, M., Lemke, R., Lenzen, R., Mandel, H., Polsterer, K., Rohloff, R.-R., Schuetze, A., Seltmann, A., Thatte, N. A., Weiser, P., and Xu, W., "LUCIFER: a Multi-Mode NIR Instrument for the LBT," in [Instrument Design and Performance for Optical/Infrared Ground-based Telescopes], Iye, M. and Moorwood, A. F. M., eds., Society of Photo-Optical Instrumentation Engineers (SPIE) Conference Series 4841, 962-973 (Mar. 2003).

[3] Dressel, L., [Wide Field Camera 3 Instrument Handbook, Version 7.0] (2015).

[4] Orban de Xivry, G., Rabien, S., Busoni, L., Gaessler, W., Bonaglia, M., Borelli, J., Deysenroth, M., Esposito, S., Gemperlein, H., Kulas, M., Lefebvre, M., Mazzoni, T., Peter, D., Puglisi, A., Raab, W., Rahmer, G., Sivitilli, A., Storm, J., and Ziegleder, J., "First Results of the Ground Layer Adaptive Optics System ARGOS," in [AO4ELT4], (2016).

[5] Busoni, L., Bonaglia, M., Borelli, J., Deysenroth, M., Esposito, S.and Gaessler, W., Gemperlein, H., Kulas, M., Lefebvre, M., Mazzoni, T., Orban de Xivry, G., Peter, D., Puglisi, A., Raab, W., Rabien, S., Rahmer, G., Sivitilli, A., Storm, J., and Ziegleder, J., "Commissioning of ARGOS at LBT: adaptive optics procedure," in $[A O 4 E L T 4],(2016)$. 
[6] Busoni, L., Bonaglia, M., Esposito, S., Carbonaro, L., and Rabien, S., "Final design of the wavefront sensor unit for ARGOS, the LBT's LGS facility," in [Adaptive Optics Systems II], Society of Photo-Optical Instrumentation Engineers (SPIE) Conference Series 7736, 77365K (July 2010).

[7] Busoni, L., Bonaglia, M., Carbonaro, L., Mazzoni, T., Antichi, J., Esposito, S., Orban De Xivry, G., and Rabien, S., "Integration and laboratory characterization of the ARGOS laser guide star wavefront sensors," in [Proceedings of the Third AO4ELT Conference], Esposito, S. and Fini, L., eds., 92 (Dec. 2013).

[8] Bonaglia, M., Busoni, L., Mazzoni, T., Puglisi, A., Antichi, J., Esposito, S., Orban de Xivry, G., and Rabien, S., "Pre-shipment test of the ARGOS laser guide star wavefront sensor," in [Society of PhotoOptical Instrumentation Engineers (SPIE) Conference Series], Society of Photo-Optical Instrumentation Engineers (SPIE) Conference Series 9148, 5 (Aug. 2014).

[9] Orban de Xivry, G., Bonaglia, M., Borelli, J., Busoni, L., Connot, C., Esposito, S., Gaessler, W., Kulas, M., Mazzoni, T., Puglisi, A., Rabien, S., Storm, J., and Ziegleder, J., "ARGOS wavefront sensing: from detection to correction," in [Society of Photo-Optical Instrumentation Engineers (SPIE) Conference Series], Society of Photo-Optical Instrumentation Engineers (SPIE) Conference Series 9148, 34 (Aug. 2014).

[10] Deysenroth, M., Honsberg, M., Gemperlein, H., Ziegleder, J., Raab, W., Rabien, S., Barl, L., Gässler, W., and Borelli, J. L., "ARGOS laser system mechanical design," in [Adaptive Optics Systems IV], Society of Photo-Optical Instrumentation Engineers (SPIE) Conference Series 9148, 91483H (July 2014).

[11] Raab, W., Rabien, S., Gässler, W., Esposito, S., Barl, L., Borelli, J., Daysenroth, M., Gemperlein, H., Kulas, M., and Ziegleder, J., "The ARGOS laser system: green light for ground layer adaptive optics at the LBT," in [Adaptive Optics Systems IV], Society of Photo-Optical Instrumentation Engineers (SPIE) Conference Series 9148, 91483K (July 2014).

[12] Peter, D., Gässler, W., Borelli, J., Barl, L., and Rabien, S., "Vibration control for the ARGOS laser launch path," in [Adaptive Optics Systems III], Society of Photo-Optical Instrumentation Engineers (SPIE) Conference Series 8447, 84474J (July 2012).

[13] Borelli, J., Barl, L., Gässler, W., Kulas, M., and Rabien, S., "Service-oriented architecture for the ARGOS instrument control software," in [Software and Cyberinfrastructure for Astronomy II], Society of PhotoOptical Instrumentation Engineers (SPIE) Conference Series 8451, 84510G (Sept. 2012).

[14] Kulas, M., Borelli, J. L., Gässler, W., Peter, D., Rabien, S., Orban de Xivry, G., Busoni, L., Bonaglia, M., Mazzoni, T., and Rahmer, G., "Practical experience with test-driven development during commissioning of the multi-star AO system ARGOS," in [Society of Photo-Optical Instrumentation Engineers (SPIE) Conference Series], Society of Photo-Optical Instrumentation Engineers (SPIE) Conference Series 9152, 0 (July 2014).

[15] Coles, W. A., Murphy, T. W., Melser, J. F., Tu, J. K., White, G. A., Kassabian, K. H., Bales, K., and Baumgartner, B. B., "A Radio System for Avoiding Illuminating Aircraft with a Laser Beam," PASP 124, 42-50 (Jan. 2012).

[16] Olivier, S. S., Max, C. E., Gavel, D. T., and Brase, J. M., "Tip-tilt compensation - Resolution limits for ground-based telescopes using laser guide star adaptive optics," ApJ 407, 428-439 (Apr. 1993).

[17] Rahmer, G. e. a., "Aircraft avoidance for laser propagation at the Large Binocular Telescope Observatory: life under a busy airspace," in [Society of Photo-Optical Instrumentation Engineers (SPIE) Conference Series], Society of Photo-Optical Instrumentation Engineers (SPIE) Conference Series 9910 (2016).

[18] Stetson, P. B., "DAOPHOT - A computer program for crowded-field stellar photometry," PASP 99, 191-222 (Mar. 1987).

[19] Mazzoni, T., Busoni, L., Bonaglia, M., and Esposito, S., "Implementation of SLODAR atmospheric turbulence profiling to the ARGOS system," in [Society of Photo-Optical Instrumentation Engineers (SPIE) Conference Series], Society of Photo-Optical Instrumentation Engineers (SPIE) Conference Series 9909 (2016). 
[20] Astropy Collaboration, Robitaille, T. P., Tollerud, E. J., Greenfield, P., Droettboom, M., Bray, E., Aldcroft, T., Davis, M., Ginsburg, A., Price-Whelan, A. M., Kerzendorf, W. E., Conley, A., Crighton, N., Barbary, K., Muna, D., Ferguson, H., Grollier, F., Parikh, M. M., Nair, P. H., Unther, H. M., Deil, C., Woillez, J., Conseil, S., Kramer, R., Turner, J. E. H., Singer, L., Fox, R., Weaver, B. A., Zabalza, V., Edwards, Z. I., Azalee Bostroem, K., Burke, D. J., Casey, A. R., Crawford, S. M., Dencheva, N., Ely, J., Jenness, T., Labrie, K., Lim, P. L., Pierfederici, F., Pontzen, A., Ptak, A., Refsdal, B., Servillat, M., and Streicher, O., "Astropy: A community Python package for astronomy," A\&A 558, A33 (Oct. 2013). 